\title{
Increased wholegrain intake does not reduce blood pressure and other markers of cardiovascular disease risk in unmedicated pre-hypertensive/ stage 1 hypertensive volunteers
}

\author{
I.G. Amadi ${ }^{1}$, K. Scott ${ }^{1}$, S. Moir ${ }^{1}$, G. Horgan ${ }^{4}$, J. Martin ${ }^{3}$, M.J. Macleod ${ }^{2}$ and F. Thies ${ }^{1}$ \\ ${ }^{1}$ Rowett Institute, School of Medicine, Medical Sciences and Nutrition, University of Aberdeen, AB252ZD, \\ ${ }^{2}$ Institute of medical Sciences, School of Medicine, Medical Sciences and Nutrition, University of Aberdeen, AB252ZD, \\ ${ }^{3}$ Biomathematics and Statistics Scotland, Rowett Institute, University of Aberdeen, AB252ZD and
}

Cardiovascular disease (CVD) morbidity is the main cause of mortality in Western countries. While poor lifestyle and unbalanced nutrition are important determinants for the disease onset, epidemiological evidence suggests that increased intake of particular dietary components, such as wholegrains, can reduce the risk of $\mathrm{CVD}^{(1)}$. The results from a previous study also showed that daily intakes of 3 portions of wholegrain foods over 12 weeks can significantly lower blood pressure in normotensive volunteers ${ }^{(2)}$. Such effect would be even more beneficial in people with non-treated elevated blood pressure. This study aimed to investigate the effects of three daily portions of wholegrain foods on markers of CVD risk in unmedicated prehypertensive/stage-1 hypertensive volunteers.

Fifty-nine volunteers ( 25 men, 34 women) underwent a 14-week cross-over randomised controlled trial. After a 2-week run in period on a refined diet, volunteers were randomly assigned to a wholegrain diet or a refined diet for 6 weeks, and then switched to the other intervention arm for 6 weeks. Ambulatory $24 \mathrm{~h}$ blood pressures (ABP), augmentation index, central aortic pressure, blood lipid and glucose concentrations were measured at baseline and after each intervention period. Differences in changes from baseline between the dietary intervention groups were analysed using two-way ANOVA $(\mathrm{p}<0.05)$.

Neither intervention significantly affected systolic ABP (pre vs post intervention values, $134.9 \pm 1.4$ vs $136.1 \pm 1.7$ and $134.1 \pm 1.6$ vs $136.0 \pm 1.5 \mathrm{~mm} \mathrm{Hg}$ for refined and wholegrains interventions, respectively).

Diastolic ABP and central blood pressures, augmentation index, blood lipids and glucose concentrations remained also unchanged.

In conclusion, daily consumption of three portions of wholegrain cereals over 6 weeks did not beneficially affect conventional risk markers for CVD in non-treated hypertensive/prehypertensive people.

1. Kirwan, JP, Malin, SK, Ross, AB et al. (2016) J Nutr 146, 2244-2255

2. Tighe P, Vaghan N, Duthie G et al. (2010) Am J Clin Nutr 92, 733-740 